\title{
DOSSIER TENS-SCL 2012 \\ LA INFLUENCIA DEL MONTAJE EN EL DISEÑO DE LAS TENSOESTRUCTURAS
}

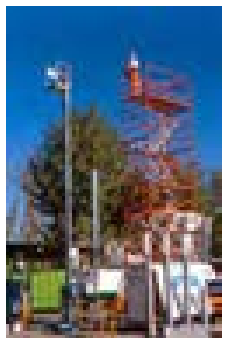

FIG 1 Medios auxiliares: plataforma autopropulsada y andamio.

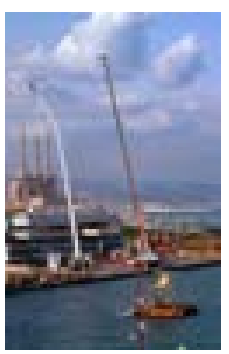

FIG 3 Medios auxiliares: plataforma autopropulsada y grúa.


FIG 4 Y FIG 5 Montaje de la carpa del circo desde el suelo.
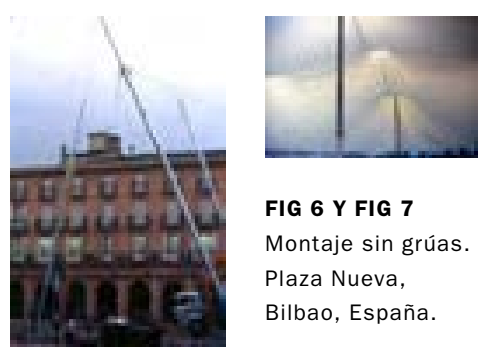

FIG 6 Y FIG 7 Montaje sin grúas. Plaza Nueva, Bilbao, España.
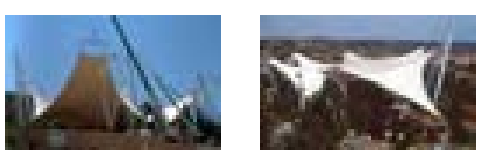

FIG 8 Y FIG 9 Montaje con grúas móviles. Camp de Mart, Tarragona, España.

\section{RESUMEN}

Las cubiertas textiles se ejecutan ensamblando la estructura de soporte y las diferentes piezas que constituyen la membrana. El proceso de montaje afecta al diseño y representa una parte importante del coste final, pero es difícil formular recomendaciones detalladas porque cada caso tiene particularidades propias. Por ello, la experiencia es muy útil ya que ayuda a resolver situaciones inesperadas y proporciona información para aplicar a los montajes siguientes.

A continuación se presenta el montaje de las tensoestructuras desde el punto de vista de su influencia en el diseño, es decir, de los aspectos del montaje a considerar en la fase de proyecto para conseguir que la cubierta se pueda construir y que el coste resultante sea razonable.

La primera parte presenta las características de la ejecución en obra, los medios necesarios para llevarla a cabo y el listado de las operaciones a considerar, junto con algunas observaciones de tipo general. Se subdivide el proceso en las fases de planificación, anclajes y cimientos, estructura de soporte, membrana, accesorios y control.

La segunda parte recoge algunos casos seleccionados en función del interés que puedan tener para proyectos posteriores. Son casos de condicionamientos de la situación y del entorno, la climatología, los medios de transporte y elevación, la calificación de la mano de obra, el plazo disponible, la estabilidad provisional de la estructura o la conveniencia de utilizar estructuras auxiliares diseñadas para la ocasión.

\section{INTRODUCCIÓN}

Las tensoestructuras están tan condicionadas por el montaje, que tanto sus principales características como los elementos y componentes, medios de elevación y transporte y operaciones de puesta en obra, deben ser tenidos en cuenta en fase de proyecto.

\section{CARACTERÍSTICAS DEL PROCESO DE EJECUCIÓN}

El tamaño y el peso de las diferentes partes, el equipo necesario para la elevación y el transporte, la accesibilidad, el equipo humano, el plazo, la seguridad y las condiciones climatológicas son aspectos muy relevantes. La forma y el pretensado resultantes del proceso de ejecución determinan no solamente el comportamiento, sino que también influyen en la resistencia y estabilidad.
El coste del montaje también es significativo. Puede representar el 30\% del presupuesto de ejecución material de las cimentaciones, la estructura, la membrana y los accesorios, por lo que se debe plantear una solución que no lo desequilibre. También se debe considerar que, después de la primera instalación, habrá que controlar las tensiones y restituirlas, así como realizar operaciones de mantenimiento en función de los materiales empleados y de la agresividad del ambiente.

\section{ELEMENTOS CONSTRUCTIVOS Y MEDIOS}

\section{AUXILIARES}

Las cimentaciones pueden ser permanentes o provisionales, anclajes superficiales o profundos, zapatas, pozos, bloques, elementos de muro pantalla o pilotes. En tanto, los elementos estructurales de soporte utilizados más a menudo son los mástiles centrales o exteriores, arcos, pórticos o las conexiones a edificios existentes.

Por su parte, la membrana requiere descarga, desplegado, presentado, montaje de cables y accesorios, costura en obra, manipulación, sujeción y tensado para lo que suelen necesitarse puntos de agarre provisionales y herramientas adecuadas. Suelen haber muchos accesorios diferentes de acuerdo al tipo (grilletes, pletinas, tornillos, tensores, sujeta cables, anillas, mordazas, manguitos y mecanismos de regulación), tamaño y resistencia que se prestan fácilmente a intercambio y confusión.

La factibilidad del proyecto depende de los medios auxiliares y, sin embargo, estos pueden variar mucho y no estar todos disponibles. Se utilizan grúas, andamios, plataformas, cestas elevadoras, gatos, cabrestantes, polipastos, cables auxiliares, herramientas y accesorios. Es frecuente que requieran puntos de sujeción provisional que no pueden coincidir con los definitivos, por lo que se tienen que incluir en el diseño inicial (figs. 1 a 3).

\section{OPERACIONES A REALIZAR}

Preparación:

1. Estudio realista y revisión del proyecto, medios disponibles y emplazamiento.

2. Programa de montaje con estudio de la estabilidad y cálculo de las fases intermedias.

\section{Cimentaciones y estructura:}

3. Replanteo.

4. Anclajes, cimentaciones y conexiones a edificios existentes.
5. Soportes estructurales fijos.

6. Posicionamiento de los elementos estructurales que se colocarán conjuntamente con la membrana.

7. Estructuras y cables auxiliares para el montaje y la estabilidad.

Membrana, cables y accesorios:

8. Control del posicionamiento de los elementos estructurales, puntos de conexión y anclaje y de la orientación de las pletinas.

9. Recepción y verificación de las características, dimensiones, tolerancias y etiquetado de los lienzos o paños, cables y accesorios de acuerdo con los planos de montaje.

10. Ejecución de bordes, esquinas y juntas.

11. Protecciones para evitar el ensuciamiento y los daños durante la ejecución.

12. Posicionamiento preliminar.

13. Levantamiento de la membrana y los soportes asociados. Suele requerir deslizamientos y rotaciones. Hay que garantizar la estabilidad durante todo el proceso.

14. Conexiones finales a los puntos y líneas de sujeción

15. Tensado. Hay varios procedimientos tales como estirar o empujar desde el exterior o el interior de la estructura, ya sea gradual o alternativamente, y de forma manual o mecánica con la ayuda de gatos, tensores o mecanismos ajustables.

16. Control de la geometría y pretensado.

17. Ajuste final.

18. Recogida de andamios y otros elementos auxiliares.

19. Inspección exhaustiva de desviaciones, manchas y defectos.

20. Entrega.

\section{OBSERVACIONES}

Las condiciones atmosféricas pueden ser disuasorias. Hay que interrumpir el montaje si la velocidad del viento supera los $10 \mathrm{~km} / \mathrm{h}$, si la temperatura es inferior a los $5^{\circ} \mathrm{C}$ o si las lluvias son muy intensas. Para estos casos hay que prever estabilidad y protección. Si la entrega se retrasa, habrá que mantener la limpieza, la seguridad e integridad evitando suciedad y daños que podrían ser atribuidos al constructor. Posterior a la entrega son necesarias operaciones de inspección, limpieza, mantenimiento del pretensado, reparación o sustitución para las que tiene que ser posible el acceso y la visibilidad. 

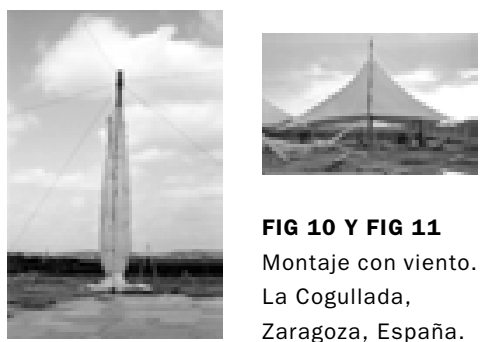

FIG 10 Y FIG 11

Montaje con viento. La Cogullada, Zaragoza, España.



FIG 12 Y FIG 13 El diseño facilita el acceso a la cubierta. Pabellón Polideportivo Municipal de Rubí, España.


FIG 14 Y FIG 15 Mástil que se articula durante el montaje y se empotra al final. Golf de Sant Cugat del Vallès, España.

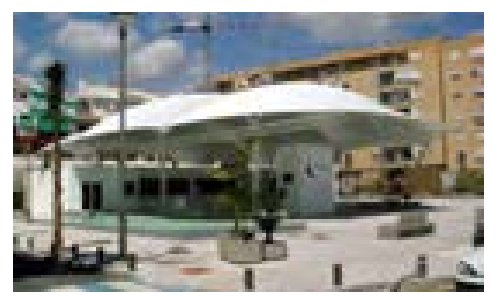

FIG 16 La cubierta del acuario de Almuñécar, España, es una pieza única de $700 \mathrm{~m}^{2}$.

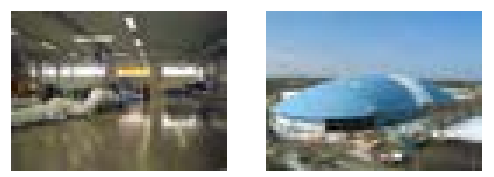

FIG 17 Y FIG 18 La cubierta presostática de Matti Orpana, Finlandia, es una pieza única de $14.200 \mathrm{~m}^{2}$.



FIG 19 Y FIG 20

Pétalos del pabellón de Venezuela en la Exposición Internacional Hannover de 2000 , montados sobre una estructura auxiliar desmontable.

\section{EL MONTAJE DESDE EL SUELO}

Las grandes tiendas tradicionales como el circo, todavía en uso, o los entoldados, ya desaparecidos, se montaban desde el suelo sin grúas ya que no podían utilizarse para este tipo de construcción. Este requerimiento obligaba a articular la base de los mástiles para levantarlos desde el suelo tirando del extremo superior. La cubierta se izaba posteriormente mediante cuerdas y poleas, y la accesibilidad tenía que ser proporcionada por la propia estructura. Estos ejemplos históricos todavía son fuente de inspiración para el montaje de las estructuras actuales (figs. 4 y 5 ).

\section{MONTAJE SIN GRÚAS}

En la actualidad, montar desde el suelo sigue siendo muy útil para los lugares a los que no se puede acceder con vehículos pesados, como por ejemplo las plazas de los cascos antiguos de las poblaciones históricas. Este fue el caso de la cubierta textil para la plaza Nueva de Bilbao en España que, al contar con distintos pórticos, solamente permite el acceso con vehículos ligeros. Los cuatro mástiles centrales articulados se levantaron desde el suelo por medio de aparatos elevadores manuales portátiles de cable pasante TIRFOR. La membrana, subdividida en cuatro partes, se sujetó a cinco coronas que se izaron con cables y poleas alojadas en los propios mástiles. No fueron necesarios andamios, grúas ni cestas elevadoras (figs. 6 y 7 ).

\section{MONTAJE CON GRÚAS MÓVILES}

El auditorio del Campo de Marte de Tarragona es un anfiteatro al aire libre con capacidad para tres mil espectadores. Se cubrió con una membrana soportada por cinco mástiles articulados y fue anclada a seis puntos bajos. Los mástiles y la membrana se tuvieron que colocar con grúas móviles desde el exterior, ya que las graderías no permitían la circulación de vehículos pesados. El tensado inicial de $300 \mathrm{kp} / \mathrm{m}$ se consiguió inclinando los mástiles hacia el exterior disminuyendo la longitud de los cables perimetrales mediante accesorios regulables (figs. 8 y 9).

La membrana de $4,800 \mathrm{~m}^{2}(7.000 \mathrm{kp})$ solamente llegó confeccionada en dos piezas de $2,400 \mathrm{~m}^{2}(3.500 \mathrm{kp})$ cada una Se tardaron cinco meses en realizar los 93 micropilotes de cimentación a $17 \mathrm{~m}$ de profundidad, dos meses en montar la estructura metálica de soporte y un mes para la cubierta.

\section{MONTAJE CON VIENTO}

Para celebrar el vigésimo quinto aniversario de la Confederación de Cajas de Ahorro de España, se construyó un pabellón efímero de $1.600 \mathrm{~m}^{2}$ en La Cogullada, cerca de Zaragoza, España. Debido al plazo disponible a fecha fija, la construcción se tuvo que realizar sin interrupciones, por lo que se tuvo que prever que estuviese sometida al fuerte viento local.

Para ello, en lugar de proyectar la cubierta en un solo lienzo, que hubiera sido imposible manipular, se proyectó fragmentada en 16 partes $u$ hojas de $10 \mathrm{x}$ $10 \mathrm{~m}$ en proyección horizontal. Cada parte era un paraboloide hiperbólico que se asociaba con otros tres, de manera que los cuatro paraboloides formaban un pseudo conoide y los cuatro pseudo conoides formaban la cubierta total alrededor de cuatro mástiles interiores, atirantada hacia el exterior por triángulos perimetrales articulados.

Para el montaje bajo la acción del viento se enrollaron en vertical las 16 hojas y se izaron desde la parte superior de los cuatro mástiles esperando el amaine. En el momento en que se produjo, se desplegaron y amarraron los puntos bajos de las 16 hojas a los triángulos perimetrales y se tensaron los bordes hasta conseguir la posición final (figs. 10 y 11).

\section{PROYECTAR FACILITANDO EL MONTAJE Y} EL MANTENIMIENTO

La pista municipal de Rubí es un equipamiento urbano al aire libre de 1.200 $\mathrm{m}^{2}$ que utilizan las escuelas, entidades deportivas y asociaciones de vecinos. $\mathrm{El}$ Ayuntamiento encargó una cubierta para evitar la lluvia y el soleamiento excesivo con la condición de que fuese muy fácil de instalar y de mantener, porque el personal que se haría cargo de ella no era especializado.

Para conseguirlo, se proyectó la estructura formada por cuatro arcos dobles sobre pilares en celosía y la cubierta subdividida en tres partes independientes que van atadas a lo largo de todo el perímetro a la estructura tubular. Los arcos son dobles para formar una pasarela accesible con el objeto de facilitar tanto las operaciones de instalación como las de mantenimiento. De este modo se satisficieron los requerimientos exigidos, porque se ha conseguido que haya protección solar y de la lluvia con una cubierta que no requiere personal especializado para su manutención (figs. 12 y 13)

\section{LAS ARTICULACIONES FACILITAN EL MONTAJE Y LOS EMPOTRAMIENTOS LA ESTABILIDAD}

Con el diseño de las uniones también se puede facilitar o complicar el montaje. Anteriormente se ha hecho referencia a la necesidad de prever puntos de sujeción provisional que, a menudo, pueden no coincidir con los definitivos. Además, el montaje es más fácil si las uniones son articulaciones regulables, mientras que, para la estabilidad definitiva, pueden interesar los empotramientos. Este es el caso de las bases de los pilares.

Las bases articuladas requieren una estabilización definitiva que puede ser proporcionada por la propia cubierta. Sin embargo, es aconsejable que este arriostramiento no se confíe solamente a la membrana porque esta es vulnerable. Por lo tanto, aparecerán unos cables o vientos inclinados de arriostramiento que pueden interferir con el uso en las zonas transitables. Esto se puede evitar empotrando la base del mástil, por lo que una solución consiste en proyectar una articulación que se bloquee al llegar a la posición definitiva.

Este fue el caso de los módulos abiertos desmontables para el campeonato de golf de Sant Cugat, España. Tenían 9 x 9 $\mathrm{m}$ y resultaba conveniente que quedaran completamente abiertos y transitables. Por ello se proyectaron apoyados sobre un único mástil central sin vientos que se acabó empotrando a unos perfiles laterales anclados en la cimentación (figs. 14 y 15)

\section{LA CONFECCIÓN DE LA CUBIERTA}

La confección de la cubierta se realiza en el taller soldando patrones. En función del tamaño de la misma y de los medios disponibles para la elevación y el transporte, se subdivide en un número mayor o menor de piezas, que se deben unir mediante juntas ejecutadas en obra. Como se trata de una operación laboriosa de la que depende en gran medida el resultado final, puede optarse por la confección de toda la cubierta en una sola pieza o lienzo.

Así se realizó la cubierta del acuario de Almuñécar, España. Tiene $700 \mathrm{~m}^{2} \mathrm{de}$ superficie, se confeccionó de una sola pieza, se apoya sobre arcos tubulares y se ancla a diez puntos perimetrales mediante mecanismos regulables que permiten ajustar la tensión (fig. 16)

El caso más espectacular es el de la cubierta presostática de Matti Orpana en Finlandia. Tiene $14.300 \mathrm{~m}^{2}$ que se confeccionaron de una sola pieza para abreviar al máximo la ejecución en obra. Se trasladó con un transporte especial y tardó dos horas en hincharse (figs. 17 y 18).

\section{ESTRUCTURAS AUXILIARES}

La organización de la obra puede obligar a construir estructuras auxiliares temporales para evitar interferencias o abreviar los plazos de ejecución. Esto sucedió en el pabellón de Venezuela proyectado por Fruto Vivas para la Expo de Hannover 200o, trasladado actualmente a Barquisimeto, Venezuela. Se trataba de una con flor de grandes dimensiones que cubría el edificio del pabellón. Como ambas construcciones debían ser simultáneas, se optó por montar el esqueleto de los pétalos sobre una estructura auxiliar y atornillarlos seguidamente al tallo central desde el exterior mediante cestas elevadoras independientes de la construcción de las plataformas interiores (figs. 19 y 20).

\section{BIBLIOGRAFÍA}

BROOKES, Alan; GRECH, Chris.

Connections. Studies in Building Assembly. Oxford, Butterworth Architecture, 1992. DE LLORENS, José Ignacio. "Details in context. Case studies". En: Marijke Mollaert. Bruselas, Universidad Vrije, 2003. FORSTER, Brian; MOLLAERT, Marijke (eds.). Tensinet Design Guide [en línea]. 2004. Disponible en <www.tensinet.com> GRÜNDIG, Lothar (ed.). Textile Roofs. Berlín, Universidad Técnica de Berlín, 1995-2013. HUNTINGTON, Craig. The Tensioned Fabric Roof. Reston, ASCE Press, 2004. $\mathrm{KOCH}$, Klaus-Michael; Habermann, Karl \& FORSTER, Brian. Membrane Structures. Munich, Prestel, 2004.

KNIPPERS, Jan. et al. Construction manual for polymers + membranes. Munich, Edition Detail, 2011.

MONJO, Juan. Introducción a la arquitectura textil. Cubiertas colgadas. Madrid, COAM, 1994.

SEIDEL, Michael. Tensile surface

structures. Berlín, Ernst \& Sohn, 2009. 